\title{
BIOÉTICA GLOBAL Y DERECHOS HUMANOS: ¿UNA POSIBLE FUNDAMENTACIÓN UNIVERSAL PARA LA BIOÉTICA? PROBLEMAS Y PERSPECTIVAS
}

\author{
Cristián Borgoño Barros*
}

\begin{abstract}
Resumen: El artículo muestra la situación actual del debate en torno a la posibilidad de fundamentar una bioética de alcance universal. Se da particular atención al intento de invocar el paradigma de los derechos humanos, exhibiendo fuerzas y debilidades de este camino, que parece haber alcanzado una cierta madurez con la adopción de la Convención de Oviedo y la Declaración Universal sobre Bioética y Derechos Humanos de la UNESCO. Se postula que es necesario reconsiderar el concepto de dignidad humana de acuerdo con la intención de los redactores de la Declaración del 48, para evitar el peligro de fragmentación y ambigüedad de este concepto.
\end{abstract}

Palabras clave: derechos humanos, bioderecho, dignidad humana, bioética global

\section{GLOBAL BIOETHICS AND HUMAN RIGHTS: A POSSIBLE UNIVERSAL FOUNDATION FOR BIOETHICS? PROBLEMS AND PERSPECTIVES}

\begin{abstract}
The article shows the current status of debate on the possibility to found a bioethics of universal reach. The article gives particular attention to the intent to invoke the paradigm of human rights, displaying the strengths and weaknesses of this option, that has appeared to have reached a certain maturity with the adoption of the Oviedo Convention and the Universal Declaration of Bioethics and Human Rights of UNESCO. It poses the necessity to reconsider a concept of human dignity which is in agreement with the original intention of the authors of the 1948 Declaration of Human Rights, in order to avoid the danger of fragmentation and ambiguity of this concept.
\end{abstract}

Key words: human rights, biorights, human dignity, global bioethics

\section{BIOÉTICA GLOBAL E DIREITOS HUMANOS: UMA POSSÍVEL FUNDAMENTAÇÃO UNIVERSAL PARA A BIOÉTICA? PROBLEMAS E PERSPECTIVAS}

Resumo: O artigo mostra a situação atual do debate em torno da possibilidade de fundamentar uma bioética de alcance universal. Uma atençáo especial ao intento de invocar o paradigma dos directos humanos, apresentando as forças de debilidades deste caminho. Este parece ter alcançado uma certa maturidade com a adoção da Convençâo de Oviedo e a Declaração Universal de Bioética e Direitos Humanos da UNESCO. Postula-se que é necesario reconsiderar o conceito de dignidade humano de acordo com a intenção dos redatores da Declaração de 48, para evitar o perigo de fragmentação e ambiguidade deste conceito.

Palavras chave: direitos humanos, biodireito, dignidade humana, bioética global 


\section{Introducción}

Hace algunos ańos, Diego Gracia mostraba en esta misma revista la tendencia de la bioética a transformarse en una bioética global(1). Su diagnóstico parece acertado si damos una mirada a los más recientes debates que, a mi juicio, se pueden encuadrar en dos filones fundamentales: "posibilista" e "imposibilista". El primero tiende a apoyarse en la fuerza retórica de los derechos humanos -como ética universal- y concentra sus esfuerzos en la búsqueda de consensos internacionales, en sedes intergubernamentales, que permitan delimitar algunos principios universalmente compartidos. Como muestra, por ejemplo, la reciente Declaración Universal sobre Bioética y Derechos Humanos de la UNESCO.

El segundo, en cambio, liderado por H.T. Engelhardt(2) denuncia la imposibilidad de un acuerdo universal entre las diversas culturas, lo que nos obligaría a abandonar de plano el proyecto mismo de la bioética global para pensar en soluciones más pragmáticas y menos comprometedoras desde el punto de vista teórico. Algunos, como Roberto Andorno(3), consideran que el problema de la fundamentación es secundario, dado que estamos delante de un consenso amplio ya establecido -por ejemplo, en las declaración de la UNESCO- que permite un esfuerzo común sobre una buena parte de los problemas de bioética.

Sin embargo, el riesgo de evitar el debate sobre la fundamentación puede llevar a la bioética global al mismo peligro que aquel que actualmente enfrentan los derechos humanos: una crisis de universalidad(4). Más aún, no es posible una adecuada tutela sobre los derechos humanos si no logramos un acuerdo mínimo sobre su fundamentación(5) y me parece que las contradicciones provocadas por la interpretación jurídica de estos textos-que hoy presenciamos-son una prueba de la intrínseca fragilidad de los derechos humanos, lo que hace pensar que estamos todavía frente a un "proyecto en construcción"(6).

Este trabajo presenta las líneas generales del debate en curso, haciendo un recorrido histórico para mostrar las características de cada uno de los filones que hemos mencionado y de qué modo, al final de cuentas, ambos deben medirse con el mismo problema: el ataque del relativismo cultural a la posibilidad de una bioética (mínima) universal.
Cuando hablo de bioética mínima pretendo poner de manifiesto que aunque el paradigma de los derechos humanos no se puede extender a todos y cada uno de los problemas de la bioética, éste constituye sin embargo un mínimo de tutela que se debe garantizar a todos los seres humanos ante el uso indiscriminado de la tecnología biomédica.

No pretendo ofrecer un modelo alternativo de fundamentación de la bioética; más bien, mostrar la viabilidad del paradigma de los derechos humanos como propuesta de fundamentación, siempre que se supere la crisis que hoy afecta al concepto que le sirve de base: la dignidad humana. Podríamos decir que ésta, como fundamento de los derechos humanos en la Declaración de 1948, ofrece ya una fundamentación suficiente que se presenta a sí misma como transcultural, aunque la globalización en curso nos obligue a retomar el debate iniciado en esos años, pues el progresivo acercamiento entre las culturas hace más patente su diversidad. La misma bioética, por otra parte, nos muestra cómo el concepto de "dignidad humana" se está fragmentando progresivamente y corre el riesgo -desgraciadamente ya sufrido por el concepto de "persona"- de transformarse en un concepto ambiguo e intrascendente(7), vaciando así de contenido la preciosa intuición de los redactores de la Declaración de 1948 y la misma idea plurisecular de dignidad humana.

\section{La bioética global: claves para el análisis de un concepto}

Hay que atribuir a V. R. Potter el origen del término "bioética global". No es este el lugar para un análisis exhaustivo del concepto potteriano, pero parece claro que es una reformulación de su concepto originario de bioética $(8,9)$, tomando en cuenta que durante sus primeros 20 años la bioética siguió un camino diverso al proyecto trazado originalmente por el oncólogo americano. La bioética global de Potter es una invitación para que la bioética retorne a su idea primitiva de ciencia de la supervivencia(10), incorporando eso sí los problemas de ética biomédica vinculados con el ideal de Potter de "supervivencia aceptable" (11) y que en su formulación inicial no tenían demasiada relevancia, como el uso del aborto y de la anticoncepción con el fin de contener el crecimiento de la población, principal amenaza para la supervivencia aceptable según Potter; o bien, la eutanasia como respuesta al problema de la calidad de vida de las personas, criterio que mide 
lo que es la supervivencia aceptable para la persona individual. Con todo, la bioética global en Potter, más que un proyecto universal -en el sentido de válido para todos-, se presenta como una respuesta particular a un problema de alcance mundial: la supervivencia aceptable en un nivel planetario. Potter no pretende imponer por la fuerza su proyecto, pero invita a todos a adherir a un credo bioético que no es materia negociable ni pretende estar abierto a visiones alternativas. Como muestra el uso del término en la literatura académica más reciente, la bioética global ha adquirido, como sucedió igualmente con el concepto de bioética, un significado bastante diverso.

Fue la tremenda aceleración del proceso de globalización, a fines de la década de los 90 , lo que inició el debate de la bioética global. En principio se comenzó a constatar que varios problemas de bioética adquirían un alcance planetario: se puede pensar en la investigación biomédica, cada vez más frecuentemente proyectada en un país del Norte pero realizada en un país del Sur; en el tráfico de órganos, que muchas veces atraviesa fronteras; en la epidemia del SIDA; en las desigualdades en términos de acceso a la salud, para limitarnos sólo a los problemas más comúnmente citados. Comenzó a configurarse lo que Peter Singer llamó Global Health Ethics(12).

Naturalmente, el contenido de una ética no son sólo los problemas que debe afrontar, sino sobre todo los principios con que debe resolverlos. Por ello, no sorprende que comenzara un debate para determinar cuál modelo de bioética era el más adecuado para solucionar estos problemas de alcance global. El primero de los candidatos, y por lo demás el más obvio, vista su influencia en la bioética norteamericana, era el principialismo(13). En realidad, para éste la bioética global no supone ningún problema adicional, pues se presenta a sí mismo como capaz, de iure, de dar respuestas universales, tanto por el carácter prima facie de los principios como por la flexibilidad para resolver eventuales conflictos entre ellos(14).

No obstante, es un hecho que en otras culturas el principialismo está lejos de ser considerado algo universal(15-17). Otros autores norteamericanos, como Robert Baker(18) y Ruth Macklin(19), comenzaron a buscar esquemas diversos de fundamentación, conscientes de que el relativismo cultural era un obstáculo serio si se quiere hablar con rigor de bioética global. Daniel Callahan(20) propone una especie de conciliación entre universalismo y particularismo, pero sin indicar con demasiada claridad cómo llegar en la práctica a una armonización compartida transculturalmente. En el último tiempo, también en la bioética norteamericana -y precisamente para dar un alcance universal-, se postula el recurso a los derechos humanos como camino para salir del propio etnocentrismo(21).

H. T. Engelhardt, por su parte, a partir de su experiencia en Asia(22,23), comienza a percibir la profundidad del problema y a inclinarse progresivamente hacia una solución radicalmente escéptica, siguiendo en cierto sentido sus propios pasos y reproponiendo a escala global su tesis de los extranjeros morales y la necesidad de llegar a un acuerdo negociado entre las personas capaces de promover sus propios intereses (24). En una escala global, sin embargo, Engelhardt tiende a dar mucho más énfasis al rol de las comunidades $y$, por tanto, a pensar en una gran diversidad de comunidades autorreferentes que se guían por estándares propios y que el Estado debe aceptar mientras no lesionen los intereses de otras comunidades y los individuos conserven la libertad de adherir o no a ellas. De ese modo, la búsqueda de una bioética global se transforma en un problema irrelevante, pues lo único que debe garantizar el Estado, y más ampliamente la comunidad internacional, es que las diversas comunidades puedan organizarse libremente para vivir conforme a sus propios principios y que los individuos no sean obligados por la fuerza a adherirse a ellas. Es lo que Engelhardt llama el "libre mercado" de las preferencias éticas(2:41). Al final de cuentas, se vuelve al viejo debate entre el cosmopolitismo liberal y el particularismo comunitario, similar aunque no idéntico al del multiculturalismo. Engelhardt es también bastante crítico con la Declaración de la UNESCO de 2005, considerándola un acuerdo vacío y carente de significado concreto(2:7). No sólo es escéptico frente a la posibilidad de la bioética global, sino que rechaza explícitamente los intentos la UNESCO en esta dirección, sin duda los más significativos desde el punto de vista del derecho internacional de los derechos humanos.

\section{Bioética y derechos humanos: el largo camino hacia la bioética global}

Hay quienes sitúan el nacimiento de la bioética en la segunda postguerra(25), y en cierto sentido tienen razón si consideramos que la bioética siempre se ha presentado a sí misma como una conciencia crítica del 
progreso científico. Ante las atrocidades de la ciencia nazi, no era posible una actitud de indiferencia y la reacción crítica quedó cristalizada en los procesos de Nuremberg y en el código homónimo, un primer intento por dar un marco jurídico a la investigación médica a escala planetaria(26). Es precisamente este afán universalista lo que asemeja a la bioética de Nuremberg con la Declaración de los Derechos Humanos del año sucesivo. Si el Código de Nuremberg fue redactado con la presunción de que se trataba de principios de alcance universal(27:3), en la Declaración es la dignidad inherente al ser humano el origen de todos los derechos concretos que después serán proclamados en el articulado(28). Por eso podemos decir, con Carlo Casini, que "los derechos humanos o son universales o no existen" (29).

Con todo, a pesar de que en el Pacto Internacional sobre Derechos Civiles y Políticos de 1966 se hablara del consenso informado como principio fundamental (en el artículo 7), hubo que esperar mucho tiempo para que bioética y derechos humanos volvieran a caminar juntos. En realidad, sólo en Europa, y a partir de la Convención Europea de Derechos Humanos de 1950, se comienza a tratar el acceso igualitario a la salud en el marco de los derechos humanos. Sabemos que en Estados Unidos, donde hay una fría acogida a los derechos económicos y sociales, no podría haberse dado una situación análoga. Durante más de 20 años el único punto de unión entre bioética y derechos humanos, y sólo al interior del derecho comunitario europeo, es el derecho a la salud.

Las cosas comienzan tímidamente a cambiar a partir de la década de los 70 , cuando la perspectiva de los derechos humanos empieza a extenderse hacia otros problemas bioéticos, como la dignidad de la fase terminal de la vida, los transplantes, la ingeniería genética, etc. Será un proceso que adquirirá una aceleración decisiva con la institución del Comité Director de Bioética del Consejo de Europa. A inicios de los 90 este comité fue el principal promotor de los textos jurídicos que abordan los problemas de bioética usando el paradigma de los derechos humanos y que se consolida definitivamente en la Convención de Oviedo, abierta a la ratificación de los estados en 1997. Se insinúa también la dimensión global del vínculo entre bioética y derechos humanos (por la apertura a la firma de la convención por parte de cualquier Estado), al menos en el plano del derecho internacional de los derechos humanos. Es notoria también la función que asume la dignidad humana como principio fundante del bioderecho en este texto $y$, en general, en el derecho comunitario europeo $(31,32)$.

Antes de hablar del salto en la relación entre bioética y derechos humanos del derecho comunitario europeo al derecho internacional tout court, fundamentalmente a través de la labor de la UNESCO, creo necesario reflexionar brevemente sobre la dinámica interna de esta relación dentro del derecho comunitario. Es innegable que sin un desarrollo del marco de los derechos humanos, impulsado por las mismas exigencias de la integración europea, este vínculo habría sido imposible, aunque también hay que atribuir esta tendencia a la mentalidad jurídica europea(33). Sophie Monnier nota además el hecho de que las normas no fueron elaboradas fundamentalmente en sede jurídica o legislativa, sino en el contexto interdisciplinar de los comités de bioética, en este caso, del Comité Director de Bioética del Consejo de Europa(34). El mismo esquema se repetirá después en la UNESCO, en la cual son los comités (el Comité Internacional de Bioética y el Comité Intergubernamental de Bioética) los que toman la iniciativa en este proceso de producción normativa.

Desde el punto de vista teórico, el aspecto crucial de este proceso es la constante referencia a la dignidad humana como fundamento de los derechos de las personas en el ámbito bioético. Se trata, evidentemente, de colocarse en continuidad con la Declaración del 48 y con ambos pactos internacionales sobre derechos humanos. Desgraciadamente, el concepto de "dignidad humana" no es unívoco y su formulación jurídica se presta para evidentes ambigüedades, que se constatan rápidamente en la existencia de propuestas normativas sustancialmente diversas y casi contradictorias que pretenden apoyarse en él (véase el debate a propósito de la Resolución 1607 de la Asamblea Parlamentaria del Consejo de Europa, que propone el acceso al aborto como derecho humano).

Otro problema latente en la relación entre bioética y derechos humanos es el tema de quién es sujeto de derechos. Ya desde 1948, cuando se renunció a especificar el reconocimiento del derecho a la vida desde la concepción, para evitar posiciones de bloqueo de países que habían legalizado el aborto (el bloque soviético, fundamentalmente), se buscó infructuosamente un acuerdo unívoco sobre este tema. Es claro que los redactores de la Declaración del 48 no pretendían excluir 
la vida prenatal de la titularidad de los derechos y por ello carece de fundamento la idea de que la expresión "nacen iguales en dignidad" del Preámbulo pretenda limitar la subjetividad jurídica a los nacidos(35). Se plantea el problema de si todo ser humano, por el sólo hecho de serlo y en cualquier etapa de su existencia, es titular de los derechos humanos. Una respuesta negativa no puede sino comprometer el carácter metajurídico de los derechos humanos, pues sustancialmente deja en manos del derecho positivo la facultad de determinar la subjetividad jurídica, vaciando de contenido in radice a los derechos humanos que son precisamente "reconocidos" (y no establecidos) por la juridicidad plasmada en texto positivo. En otras palabras, su carácter vinculante no se deriva sustancialmente del hecho de su positivización.

El problema de la naturaleza declarativa (su exigencia intrínseca de reconocimiento) de los derechos humanos encierra una ambivalencia estructural que es necesario tener en cuenta. Por una parte, es necesario que los derechos humanos reciban una positivización, para lo cual es indispensable el recurso al consenso político concreto; por otra, sin embargo, este mismo proceso de positivización, exigido por la naturaleza propia de los derechos humanos, encierra siempre el peligro de reducirlos a su dimensión puramente positiva. Los derechos humanos son principios ético-jurídicos, más aún, el núcleo mismo de toda juridicidad(36). Por eso, si los despojamos de su valor ético objetivo y universal no tiene ningún sentido proponerlos como fundamento de una bioética global.

\section{Ambigüedades en torno al concepto de dignidad humana}

Como señala D'Agostino, la dignidad -concepto clave para la ética, el derecho y la misma bioética(37)- es también considerada particularmente ambigua(7). En realidad, esta ambigüedad es más patente en la reflexión bioética que en la biojurídica, lo cual expresa también la dualidad de planos de la dignidad humana: como principio ético y como principio jurídico. En este segundo caso, la dignidad humana siempre debe ser entendida dentro del contexto del documento en que se encuentra y, por lo tanto, con un significado jurídico preciso(38-40). Buena parte de la ambigüedad del concepto deriva de la diversidad de sus raíces -estoicas, bíblicas, renacentistas y liberales- que últimamente han confluido en la idea contemporánea de dignidad, la que precisamente, como muestra la elaboración de la Declaración del 48, es un concepto que aspira a servir de puente para el diálogo transcultural.

En cambio, si queremos hablar de la dignidad humana como concepto ético podemos intentar una delimitación de su contenido con el fin de darle un valor fundante. Podemos afirmar que se trata de un atributo esencial de los seres humanos, del cual se derivan consecuencias normativas tanto de índole prohibitiva (abstenerse de determinados actos contra los portadores de dignidad) como de índole prescriptiva (actuar a favor de las personas).

En el debate actual, no sólo bioético, tienden a distinguirse dos enfoques sobre la dignidad $(41,42)$ : uno que podríamos llamar ontológico y otro funcional. El primero consiste básicamente en reconocer valor normativo a la pertenencia a un conjunto de seres de igual entidad ontológica, en este caso, la especie humana. Basta la sola pertenencia a esta especie para ser inmediatamente portador de una dignidad. Este es claramente el sentido que el adjetivo "inherente" pretende dar al sustantivo "dignidad" en el preámbulo de la Declaración y, en general, en los documentos de bioderecho se tiende a seguir esta interpretación si se considera que los sujetos incapaces de autodeterminarse son también considerados sujetos de derecho.

La interpretación funcional de la dignidad humana, con algunas variantes, consiste en fundarla en una capacidad, normalmente la autodeterminación, y por lo mismo en excluir a todo aquel que no es capaz de autodeterminarse aún (vida prenatal o también neonatal) o ha perdido esta capacidad (por ancianidad o enfermedad) o no la adquirirá nunca (discapacidades graves). Esto no implica necesariamente que se pueda disponer de modo arbitrario de estas categorías de seres humanos, pero evidentemente no se les concede el estatuto de sujeto de derechos, en cuanto sus intereses quedan a merced de los intereses de otros. Esta interpretación de la dignidad no sólo puede ser gravemente discriminatoria, sino también contrasta claramente con la progresiva tendencia a la extensión de la titularidad de los derechos humanos que va desde la abolición de la esclavitud y se prolonga hasta la reciente Convención sobre la Discapacidad, en la cual paradójicamente algunos quieren conceder a los discapacitados, bajo el camuflaje de la salud reproductiva, el mismo derecho por el cual ellos son eliminados antes de nacer a través del aborto eugenésico. Afirmación 
fundamental de esta corriente $(43,24)$ es la distinción filosófica, pero con consecuencias jurídicas concretas, entre persona y ser humano, que para un enfoque ontológico de la dignidad resulta intolerable. Como decíamos, esta interpretación de la dignidad humana no es tan relevante en el Derecho Internacional, en cuanto no existe ningún texto que la abrace explícitamente. Sin embargo, puede ser particularmente importante en la legislación interna, en la que los principios del derecho internacional tienen que ser aplicados. En un plano ético, en un proyecto que busque fundamentar la bioética en los derechos humanos, la pregunta por el sujeto de los mismos no puede quedar sin respuesta. Lo claro es que las diversas concepciones del concepto de "dignidad" son dependientes de una perspectiva antropológica concreta(44).

\section{La crisis de universalidad de los derechos humanos}

Hoy es frecuente escuchar afirmaciones como que los derechos humanos son un artefacto occidental, una reliquia de la Ilustración o bien una sutil justificación del imperialismo cultural de Occidente. No se trata de una objeción totalmente nueva. En 1947, la misma American Anthropological Association criticaba el proyecto de la Declaración de los Derechos Humanos negando la universalidad de los valores a favor de un acendrado relativismo cultural(45). Últimamente, en formas más sutiles, esta objeción se ha revitalizado, no sólo por motivos religiosos (basta pensar en las dificultades del Islam con los derechos humanos) sino principalmente culturales. Esta postura es evidente en la Declaración de Bangkok(46) y en algunos aspectos de la Conferencia Mundial sobre Derechos Humanos de Viena de 1993, en la que se habla de la necesidad de contextualizarlos. En Viena, sin embargo, se llegó a una fórmula de acuerdo para afirmar su universalidad: "Todos los derechos humanos son universales, indivisibles e interdependientes. La comunidad internacional debe tratar al conjunto de los derechos humanos de modo justo y equitativo, poniéndolos al mismo nivel y dando el mismo énfasis a cada uno. Aunque se debe tomar en cuenta el significado de las particularidades nacionales y regionales, así como los diversos contextos históricos, culturales y religiosos, es un deber de los estados, más allá de sus sistemas políticos, económicos o culturales, el promover los derechos humanos y las libertades fundamentales" $(47, n .5)$.
Una concepción individualista de los derechos humanos, centrada en la autodeterminación, como la que mostramos a propósito de la dignidad humana, hiere las susceptibilidades de culturas de matriz fuertemente comunitaria, como la oriental o la africana $(48,16)$. De este modo, el clima de diálogo intercultural y de sincero esfuerzo por encontrar una vía conjunta tiende a ser reemplazado por uno de hostilidad y desconfianza, como se pudo ver hace algunos ańos en las Conferencias de El Cairo y Beijing. No cabe duda que, como señala Glendon(49), esta tendencia a la inflación de los derechos humanos en clave individualista no contribuye a un consenso universal en torno a los mismos.

Ante este escenario, la comunidad académica parece estar dividida. Mientras unos hablan de la necesidad de superar el paradigma de los derechos humanos para construir una etnobioética(50), otros, sobre todo quienes colaboran en el proyecto de la UNESCO, tienden a ser más optimistas(51). El problema de cómo lograr acuerdos sobre principios de bioética con alcance global que pudieran mediar entre diversas culturas estaba sumamente presente en la mente de los miembros del Comité de Bioética de la UNESCO cuando dieron su parecer sobre la posibilidad de elaborar "normas universales de bioética” (52). Probablemente, este problema ha estado mucho más presente en la Declaración de 2005 que en las precedentes sobre el genoma humano y la protección de los datos genéticos humanos, de 1997 y 2003 respectivamente $(53,54)$.

En realidad, más allá de la diversidad de las posiciones, es claro que se trata de un asunto que debe ser abordado. Como acabamos de ver, el problema de la universalidad transcultural de la bioética se plantea tanto si se reflexiona a un nivel estrictamente ético como si se busca un modo concreto de encontrar una universalidad compartida, como son los derechos humanos. El artículo 12 de la Declaración de la UNESCO de 2005 que afirma que: "Se debería tener debidamente en cuenta la importancia de la diversidad cultural y del pluralismo. No obstante, estas consideraciones no habrán de invocarse para atentar contra la dignidad humana, los derechos humanos y las libertades fundamentales o los principios enunciados en la presente Declaración, ni tampoco para limitar su alcance" (55), plantea el problema desde una perspectiva que tiende a contraponer la diversidad cultural a los derechos humanos, como si estos últimos fueran principios atemporales e inmutables, sin una dimensión de his- 
toricidad. Sólo si se crea una cultura de los derechos humanos, estos serán efectivamente respetados; por ello, es necesario que se revistan del ropaje que cada cultura les ofrece.

Si pensamos en los refranes de las diversas lenguas, constatamos que con expresiones literalmente diferentes se busca transmitir una misma idea o principio. Todo traductor sabe que es imposible traducir literalmente un refrán; es necesario conocer los equivalentes de cada lengua. El respeto de la diversidad cultural no es relativismo cultural, pero no porque exista un límite externo que serían los derechos humanos, sino porque existe una matriz común a todas las culturas, lo humano en su sentido más profundo, que debe ser siempre respetado y que es la medida que permite juicios transculturales. Tematizar con profundidad esta dialéctica entre lo que podríamos llamar "naturaleza" y "cultura" excede el espacio de este artículo, pero me interesa señalar que los derechos humanos, sobre todo en su formulación positiva, deben situarse al interior de una determinada cultura y no fuera de ella, en un espacio abstracto y atemporal.

\section{Un camino de salida a la crisis de universalidad de los derechos humanos}

Mary Ann Glendon señala con acierto que debemos volver nuestra mirada nuevamente al espíritu de la Declaración del 48 para elaborar lo que en ella está virtualmente contenido(28). No es irrelevante que el texto fundacional de los derechos humanos globales haya sido el fruto de una colaboración eficaz entre diversas sensibilidades culturales, como han mostrado todas las reconstrucciones del proceso de redacción desde las más remotas(56) hasta las más recientes(35). Si la analizamos no como una lista de derechos sin principio unificador sino como un conjunto armónico y bien estructurado(57), seremos capaces de desarrollar el contenido que se quiso expresar con la idea de dignidad humana inherente. Está claro que lo que se tenía en mente no era un ideal de ser humano abstracto, atemporal y fuera de la historia, sino hombres y mujeres concretos a los cuales era necesario reconocer un conjunto de derechos, teniendo en cuenta la diversidad concreta en que desarrollan su existencia. Tutelar la dignidad humana no se reduce únicamente -y ni siquiera principalmente- a garantizar que cada uno pueda hacer con su libertad lo que le parezca, sino que también implica crear un espacio para que el ser humano pueda realizarse conforme a su propia potencialidad y colaborar a proteger la dignidad de los demás, especialmente de los más débiles. Este es el espacio que quieren crear quienes promueven la tutela de los derechos humanos con la bioética en un lugar fundamental, dado que tocan la raíz del ser humano: la vida humana física como fundamento indispensable de nuestro ser en el mundo.

Es cierto que analizar este principio para llegar a principios o normas concretas en materia de bioética no es una empresa fácil (lo demuestra el mismo proceso de elaboración de la Declaración de la UNESCO) pero si se hace buscando siempre el respeto a la humanidad común que está en la base de nuestra dignidad, y que necesariamente se expresa en una cultura concreta, podemos estar confiados en que se descubrirán la exigencias específicas que se derivan de ella en el campo de la bioética. Este puede ser un punto de partida sólido para una bioética global transcultural. La Declaración de la UNESCO puede ser un buen inicio para este proyecto, si se mantiene en el camino trazado por el documento fundacional del paradigma contemporáneo de los derechos humanos: la Declaración del 48, de la que acabamos de celebrar el sexagésimo aniversario.

\section{Referencias}

1. Gracia D. De la bioética clínica a la bioética global: treinta años de evolución. Acta Bioethica 2002; 8(1): 27-39.

2. Engelhardt Jr. HT, (ed). Global bioethics. The collapse of consensus, Salem: M \& M Scrivener Press; 2006.

3. Andorno R. Comment concilier une bioéthique universelle et le respect de la diversité culturelle? In Byk C. Bioéthique et droit international. Autour de la Déclaration universelle sur la bioéthique et les droits de l'bomme, París: LexisNexis; 2007: 55-60.

4. Glendon MA. Rights Babel: The universal rights idea at the dawn of the third millennium. Gregorianum 1998; 79(4): 611-24.

5. Cotta S. Soggetto umano. Soggetto giuridico. Milan: Giuffrè; 1997.

6. Glendon MA. Foundations of human rights: The unfinished business. American Journal of Jurisprudence 1999; 44: $1-14$. 
7. Macklin R. Dignity is a useless concept. British Medical Journal 2003; 327:1419-1420.

8. Whitehouse P. Van Rensselaer Potter: the original bioethicist. Hastings Center Report 2001; 31(6): 12.

9. Russo G, Potter VR. L'idea originaria di bioetica, Itinerarium 1994; 2: 11-25.

10. Potter VR. Bioethics. The science of survival. Perspectives in Biology and Medicine 1970; 14: 127-153.

11. Potter VR. Global Bioethics. Building on the Leopold Legacy. East Lansing: Michigan State University Press; 1988: 51.

12. Singer P. Medical ethics. British Medical Journal 2000; 321: 282-285.

13. Beauchamp T, Childress J. Principles of Biomedical Ethics $5^{\text {th }}$ ed. Nueva York: Oxford University Press; 2001.

14. Beauchamp T. The mettle of moral fundamentalism. A reply to Robert Baker. Kennedy Institute of Ethics Journal 1998; 8(4): 389-401.

15. Tan Alora A, Lumitao JM, (eds.) Beyond a western bioethics. Voices from the developing world. Washington D.C.: Georgetown University Press; 2001.

16. Tangwa G. Globalization or westernization? Ethical concerns in the whole bio-business. Bioethics 1999; 13(3-4): 218226.

17. Fan R. Bioethics: Globalization, communization or localization? In Global bioethics. The collapse of consensus. Salem: M \& M Scrivener Press; 2006: 271-99.

18. Baker R. A theory of international bioethics: the negotiable and the non-negotiable. Kennedy Institute of Ethics Journal 1998; 8(3): 233-274.

19. Macklin R. Against Relativism. Cultural diversity and the search for ethical universals in medicine. Nueva York: Oxford University Press; 1999.

20. Callahan D. Universalism and particularism. Fighting to a draw. Hastings Center Report 2000; 30(1):37-44.

21. Annas GJ. Bioethics and human rights. Hastings Center Report 2003; 33(5): 3.

22. Engelhardt Jr. HT. From Western to Filippino Bioethics, An acknowledgement in gratitude for having been a colleague in a marvelous intellectual and moral journey. In Tan Alora A, Lumitao JM, (eds.) Beyond a western bioethics. Voices from the developing world, Washington D.C.: Georgetown University Press; 2001: xi-xiv.

23. Engelhardt HT. Japanese and Western Bioethics: Studies in moral diversity. In Hoshino K. (ed.) Japanese and Western Bioethics. Studies in moral diversity. Dordrecht: Kluwer; 1997: 1-10.

24. Engelhardt Jr. HT. The Foundations of Bioethics $2^{\text {nd }}$ ed. Nueva York: Oxford University Press; 1995.

25. Baker R. Bioethics and Human Rights. A Historical Perspective. Cambridge Journal of Healthcare Ethics 2001; 10: 241-252.

26. Taylor T. The anatomy of the Nuremberg trials. Nueva York: Alfred A. Knopf; 1992.

27. Annas GJ, Grodin MA, (eds.) The Nazi doctors and the Nuremberg Code. Human rights in human experimentation. Nueva York: Oxford University Press; 1992.

28. Glendon MA. A world made new. Eleanor Roosevelt and the Universal Declaration on Human Rights. Nueva York: Random House; 2001.

29. Casini C. Intervento di sintesi. In: Sgreccia E, Calabrò GP (eds.) I diritti della persona nella prospettiva bioetica e giuridica. Atti del Convegno. Lungro di Cosenza: Marco 2002: 87-95.

30. Bompiani A. Il sistema della Convenzione sui Diritti Umani e la Biomedicina del Consiglio díEuropa. La Convenzione, i Protocolli addizionali e le Raccomandazioni inerenti: un esempio di Biodiritto Europeo (en prensa).

31. Duprat JP. Le biodroit, une phénomène global sans principe unificateur? Journal International de Bioéthique 2004; 15(2-3): $37-50$

32. Andorno R. La dignidad humana como fundamento de la Bioética y de los Derechos Humanos en la Declaración Universal. In Gros-Espiell H, Gómez Sánchez Y, (eds). La Declaración Universal sobre Bioética y Derechos Humanos de la UNESCO. Albolote (Granada): Comares; 2006: 253-70.

33. Hottois G. Droits de l'homme. In Hottois G, Missa JN. Nouvelle encyclopédie de bioéthique. Bruselas: De Boeck Université; 2001: 315-321.

34. Monnier S. Les comités diéthique et le droit. Éléments dianalyse sur le système normatif de la bioétique. París: L'Harmattan; 2006.

35. Morsink J. The Universal Declaration on Human Rights. Origins, draft and intent. Filadelfia: University of Pennsylvania Press; 2000. 
Bioética global y derechos humanos: ¿una posible fundamentación universal para la bioética? - Cristián Borgoño Barros

36. D'Agostino F. Lezioni di filosofia del diritto. Turín: Giappichelli; 2006.

37. D'Agostino F. Bioetica e dignità dell'essere umano. In Mazzoni CM, (ed.) Un quadro europeo per la bioetica? Florencia: Leo S. Olschki; 1998: 153-157.

38. Andorno R. Dignity of the person in the light of international biomedical law. Medicina e Morale 2005; 1: 165-78.

39. Schmidt H. Whose dignity? Resolving ambiguities in the scope of 'human dignity' in the Universal Declaration on Bioethics and Human Rights. Journal of Medical Ethics 2007; 33: 578-584.

40. Simon J. La dignidad humana como principio regulador en bioética. Revista de Derecho y Genoma Humano 2000; 13: 25-39.

41. Aschroft RE. Making sense of dignity. Journal of Medical Ethics 2004; 31: 679-682.

42. Kilner JF. Human dignity. In Post SG, (ed.) Encyclopedia of Bioethics $3^{\text {rd }}$ ed. Londres: MacMillan; 2003: 1193-2000.

43. Singer P. Rethinking life and death. The collapse of our traditional ethics. Nueva York: St. Martin's Press; 1995.

44. Schulman A. Bioethics and the question of human dignity. In: The President's Council on Bioethics. Human dignity and bioethics. Washington D.C.: The President's Council on Bioethics; 2008: 3-18.

45. American Anthropological Association. Statement on human rights. American Anthropologist 1947; 49: 539.

46. Final Declaration of the Regional Meeting for Asia of the World Conference on Human Rights, Bangkok 1993.

47. Conferencia Mundial sobre los Derechos Humanos. Declaración de Viena y Programa de Acción, № 5. Viena: 1993.

48. Sakamoto H. Towards a new 'Global Bioethics'. Bioethics 1999; 13(3-4):191-197.

49. Glendon MA. La visione dignitaria dei diritti sotto assalto. In: Antonini L. Il traffico dei diritti insaziabili. Soveria Mannelli: Rubbettino; 2007: 59-80.

50. Byk C. Bioéthique et culture. In: Byk C. Bioéthique et droit international. Autour de la Déclaration universelle sur la bioéthique et les droits de líhomme. París: LexisNexis; 2007: xix-xx.

51. Ten Have H. The activities of UNESCO in the area of ethics. Kennedy Institute of Ethics Journal 2006; 16(4): 333351.

52. Comité Internacional de Bioética. Towards a Declaration on Universal Norms on Bioethics, Paris; 2004. Sitio en Internet. Disponible en: http://portal.unesco.org/shs/en/files/5997/10920551251rappExtraCIB_en.pdf/rappExtraCIB_en.pdf

53. Gros-Espiell H. Introduction. In: Division of Ethics of Science and Technology. Birth of the Universal Declaration on the Human Genome and Human Rights. Paris: UNESCO; 1999: 1-7.

54. Lenoir N, Mathieu B. Les normes internationales de la bioéthique 2 ed. Paris: PUF; 2004.

55. Asamblea General de la UNESCO. Declaración Universal sobre Bioetica y Derechos Humanos. París: 2005.

56. Verdoot A. Naissance et signification de la Declaration Universelle des Droits de l'Homme. Louvain: E. Warny; 1964.

57. Novak M. Human dignity, human rights. First Things 1999; 97: 39-42.

Recibido: 2 de febrero de 2009

Aceptado: 19 de febrero de 2009 